\title{
Effects of cluster location and cluster distribution on performance on the traveling salesman problem
}

\author{
James N. MacGregor ${ }^{1}$
}

Published online: 14 May 2015

(C) The Psychonomic Society, Inc. 2015

\begin{abstract}
Research on human performance in solving traveling salesman problems typically uses point sets as stimuli, and most models have proposed a processing stage at which stimulus dots are clustered. However, few empirical studies have investigated the effects of clustering on performance. In one recent study, researchers compared the effects of clustered, random, and regular stimuli, and concluded that clustering facilitates performance (Dry, Preiss, \& Wagemans, 2012). Another study suggested that these results may have been influenced by the location rather than the degree of clustering (MacGregor, 2013). Two experiments are reported that mark an attempt to disentangle these factors. The first experiment tested several combinations of degree of clustering and cluster location, and revealed mixed evidence that clustering influences performance. In a second experiment, both factors were varied independently, showing that they interact. The results are discussed in terms of the importance of clustering effects, in particular, and perceptual factors, in general, during performance of the traveling salesman problem.
\end{abstract}

Keywords Visual perception · Spatial cognition · Perceptual organization

Dot arrays have been used as stimuli for the study of grouping and clustering effects in perception at least since Wertheimer's (1923/1950) pioneering analysis. Since then, dot arrays have been used to investigate a wide variety of perceptual phenomena, including perceptual organization (Trick \& Enns, 1997),

James N. MacGregor

jmacgreg@uvic.ca

1 School of Public Administration, University of Victoria, Victoria, British Columbia V8W 2Y2, Canada clustering (O'Callaghan, 1974), depth perception (Julesz, 1965), texture perception (Pickett, 1964), apparent motion (Ramachandran \& Anstis, 1986), and the perception of biological movement (Johansson, 1973). Recently, random and patterned point sets have been used to study human performance in combinatorial optimization problems such as the traveling salesman (TSP), minimum spanning tree, and generalized Steiner tree problems (Vickers, Mayo, Heitmann, Lee, \& Hughes, 2004). Although the names may imply that such research falls in the domain of problem solving, theoretical developments and empirical research in the area have frequently cited perceptual mechanisms, and the initial studies were often published in perception journals (Lee \& Vickers, 2000; MacGregor \& Ormerod, 1996; MacGregor, Ormerod, \& Chronicle 1999; Ormerod \& Chronicle, 1999; Vickers, Butavicius, Lee, \& Medvedev, 2001). More recently, this new area of research has captured the interest of cognitive scientists working in clinical neuroscience, comparative psychology, decision making, memory, and vision (MacGregor, 2012), but often from a perception perspective. The present article continues this vein of exploring perceptual influences on TSP performance, by investigating the effects of dot clusters and their locations on human performance of the TSP. Before describing the experiments, however, a brief background summary of relevant TSP research is provided.

The TSP is one of a class of combinatorial optimization problems whose problem space typically increases exponentially with increasing number of problem elements. In the case of the planar TSP, the goal is to find the shortest Euclidean path (tour) that passes through a set of points in the plane, returning to the starting point, and the number of possible solutions is determined by $(n-1) ! / 2$, where $n$ is the number of points. As the problem size increases above a handful, solving by enumerating all possible solutions quickly becomes infeasible, even for supercomputers. For example, the 
famous Proctor \& Gamble contest to find the shortest route through 33 US cities (Applegate, Bixby, Chvátal, \& Cook, 2006 ) involved $1.32 \times 10^{35}$, or some 132 billion trillion trillion, possible routes (MacGregor \& Chu, 2011). The TSP is one of a class of problems of profound theoretical significance in mathematics and computer science (Garey \& Johnson, 1979; Lawler, Shmoys, Rinnooy Kan, \& Lenstra, 1985). It is also of considerable practical relevance in many scientific and industrial applications, including X-ray diffraction (Bland \& Shallcross, 1987), warehousing (Dallari, Marchet, \& Ruggeri, 2000), circuit board drilling (Sangalli, 1992), and the laying of ducting (Krolak, Felts, \& Marble, 1971). Because no general analytical procedure is guaranteed to find the optimal path, there has been longstanding interest in developing heuristic procedures for generating good approximate solutions.

Heuristic procedures generally require on the order of $n^{3}$ calculations (Golden, Bodin, Doyle, \& Stewart, 1980), and therefore the average processing time increases as a polynomial function of problem size. Perhaps surprisingly, human solvers have been found to outperform a number of such heuristic procedures (Hill, 1982; MacGregor \& Ormerod, 1996). More surprisingly, given the heuristics' processing requirements, human solution times appear to increase only linearly with problem size (Graham, Joshi, \& Pizlo, 2000). This trend holds for problems up to $n=120$, the largest size tested to date, whereas performance quality decreases linearly over the same range, from an average tour length of $1 \%$ above the optimal length for $n=10$, to $11 \%$ for $n=120$ (Dry, Lee, Vickers, \& Hughes, 2006). Solutions of this quality depend, however, on the problems being presented as a spatial array of dots, and if they are presented as a table of internode distances, performance is poorer (Gärling, 1989; Polivanova, 1974). These basic findings have led to suggestions that completing spatially presented TSPs is in some sense "natural" to the human perceptual system (MacGregor \& Ormerod, 1996; Ormerod \& Chronicle, 1999; Vickers, Lee, Dry, Hughes, \& McMahon, 2006). This observation is consistent with the finding that when invited to connect an array of dots so as to illustrate the pattern that they perceive, participants often draw the shortest path (Pomerantz, 1981). It may also be consistent with the proposal that human perception of clusters in dot arrays involves minimum spanning tree solutions (Zahn, 1971). A number of heuristics can find TSP solutions by first solving the minimum spanning tree (Asano, Bhattacharya, Keil, \& Yao, 1988; Lawler et al., 1985), suggesting the possibility that similar fundamental processes may underlie the perception of dot clusters, minimum spanning tree solving, and the construction of TSP solutions.

Cluster perception plays a central role in most current theories of human TSP performance (Best, 2005; Kong \& Schunn, 2007; Pizlo, Stefanov, Saalweachter, Haxhimusa, \& Kropatsch, 2006; Vickers, Lee, Dry, \& Hughes, 2003).
However, in spite of its theoretical prominence, clustering has received little empirical attention in human TSP research (MacGregor, 2013). One early study (Hirtle \& Gärling, 1992) varied the degree of clustering of the nodes, and a descriptive reanalysis of the results indicated that solutions were generally better when the stimuli were clustered (MacGregor \& Chu, 2011). MacGregor, Ormerod, and Chronicle (1999) used stimuli with 12 nodes in which a cluster of four nodes was located either centrally or near the boundary of the dot arrays, and they found that the solutions were twice as likely to be optimal when the cluster was located near the boundary.

Dry, Preiss, and Wagemans (2012) used fifteen 49-node point sets, five each at three different levels of clustering, to test the hypothesis that clustering facilitates TSP performance. To measure the degree of clustering, they used the ClarkEvans index of aggregation, $R$ (Clark \& Evans, 1954). The index is based on the average minimum distance of each node from its nearest neighbor and is constructed so that random dot clusters, on average, will have $R=1$. As dots become more clustered, $R$ decreases below 1 , whereas as dots become more regularly spaced, $R$ increases above 1 . Dry et al. (2012) used stimuli corresponding to highly clustered, random, and highly regular dot distributions, and measured participants' TSP performance in terms of tour length, as a percentage above the optimal (PAO), and solution time. The results supported the hypothesis, in that tour lengths decreased significantly with decreasing $R$ values. Solution times also decreased, although differences between the conditions were not significant.

Although the results were consistent with the hypothesis that clustering facilitates TSP performance, MacGregor (2013) suggested that another factor could have influenced the results. Because of the method of generating the stimuli of different $R$ levels, it appeared possible that a disproportionate number of clusters may have tended to fall near the perimeter of the dot arrays (the convex hull). Given the findings of MacGregor et al. (1999), the location of nodes as well as the degree of clustering may have affected performance. As an initial test of this possibility, an experiment was conducted in which a salient ten-node cluster was located either near the center of point sets or close to the boundary, while holding the $R$ value constant. The results showed that TSP tours were significantly shorter and faster when a cluster was located near a boundary rather than at the center. However, although the outcome showed that the locations of clusters do affect performance independently of the degree of clustering, it did not determine whether the degree of clustering plays a role at all, or whether the Dry et al. (2012) results arose simply because cluster location and $R$ values were confounded. Thus, the question of whether $R$ value has an effect on TSP performance independently of cluster location remains open. The present experiments were conducted to clarify this issue, 
by further examining the effects of cluster location and cluster distribution.

\section{Experiment 1}

In the reported experiment, MacGregor (2013) varied the location of clusters while holding the degree of clustering, as measured by $R$ value, constant. The present Experiment 1 was based on four conditions in which both cluster location and $R$ value were varied. Comparisons between the conditions allowed for a number of tests of the location-of-cluster and degree-of-clustering explanations. Also, MacGregor (2013) found that adding a cluster of ten nodes to the center of a random point set, but not to the periphery, resulted in poorer solutions than those to the random point set alone. However, because adding a cluster to the center also increased the number of nodes, it was unclear whether this effect was due to the location of the cluster or to the increase in the number of nodes. Experiment 1 addressed this question, also.

The experiment used 30-node point sets generated to produce four different conditions: (1) random, (2) center clusters, (3) clusters at a single edge, and (4) clusters at multiple edges (as is illustrated in Fig. 1). The mean $R$ values for the four conditions were $1.13,0.98,1.01$, and 0.75 , respectively. On the basis of Dry et al.'s (2012) findings, we would expect performance in Condition 4 to be better than that in the other three conditions, on the basis of $R$ value. The $R$ values among the three remaining conditions might be too similar to result in performance differences, but should any occur, the prediction based on $R$ value would be that performance should be better in both Conditions 2 and 3 than in Condition 1. That is, on the basis of the $R$-value explanation, we would expect the patterns Condition $4>$ Conditions 1, 2, 3 and, more tentatively, 2, $3>$ 1. On the basis of the cluster location hypothesis, the expectation would instead be that $4,3>2,1$. Finally, if the previous finding holds that centrally located clusters present a more difficult problem than a random stimulus, we would expect that Condition $1>2$. Note that because the random stimuli had somewhat larger $R$ values than the stimuli with central clusters, the potential effects of $R$ ran counter to this experimental hypothesis.

\section{Method}

Participants The participants were 20 volunteers recruited from the campus community at the University of Victoria. They were each given a $\$ 20$ cafeteria voucher for participating. A sample size of 20 was chosen because previous studies had shown effects of cluster location (MacGregor, 2013, $n=20$ ) and cluster distribution (Dry et al., 2012, $n=25$ ) using samples of this order. In addition, a power analysis based on effect sizes for PAO that was reported in Dry et al. (2012) indicated that $n=19$ should be sufficient to detect a difference.

Stimuli Five point sets were generated randomly, and each was used as the basis for producing three additional point sets, one with clusters located centrally, one with the same clusters located close to a boundary arc, and one with the clusters distributed among multiple boundary arcs. One of the five stimulus sets is illustrated in Fig. 1, which shows from left to right the original random point set, followed by the center, single-edge, and multi-edge cluster stimuli. There were 20 point sets in total, each consisting of 30 nodes. The stimuli were created in the following way.

First, a 30-node point set was randomly generated to fall within a square with sides extending from 0 to 510 pixels, subject to the constraint that no point was closer than 15 pixels to another. This was the random stimulus.

Next, to generate the cluster conditions, all but the boundary points were removed from the random stimulus, leaving $h$ nodes on the convex hull. Then the numbers and sizes of the clusters were randomly determined to provide from four to six clusters, each consisting of three to five nodes, such that the total number of nodes in clusters equaled $30-h$. Next, the relative positions of the nodes within each cluster were randomly determined, such that the minimum internode distance $\geq 5$ pixels and the maximum diameter of a cluster $\leq 40$ pixels $(1.5 \mathrm{~cm})$. The resulting means were 3.96 for cluster size, 5.20 for number of clusters, and 9.40 for number of nodes on the convex hull.

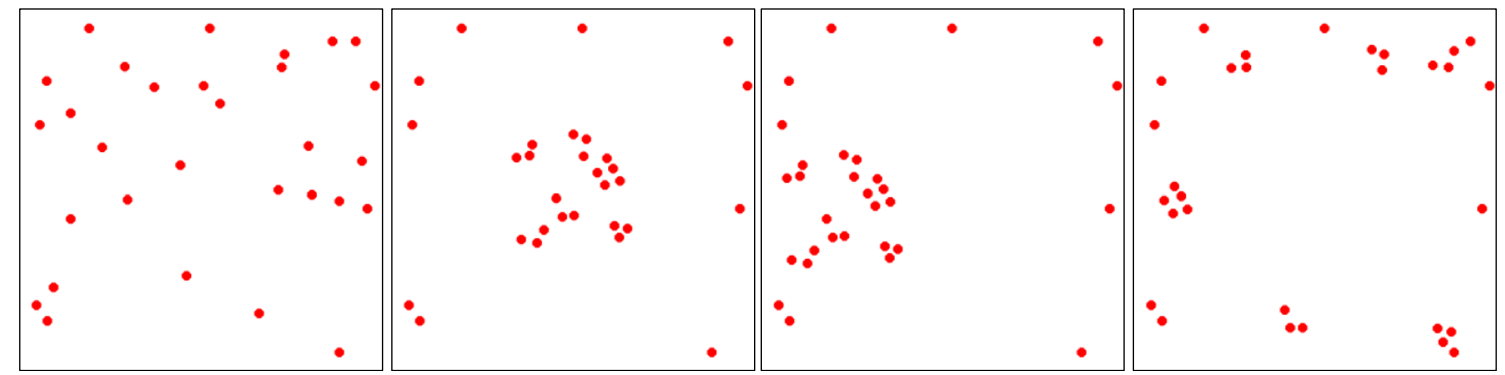

Fig. 1 Sample of TSP instances used in Experiment 1, showing, from left to right, examples of random, center-cluster, single-edge-cluster, and multiedge-cluster stimuli 
To locate clusters within the convex hull, the display was partitioned into three areas-center, intermediate, and boundary - on the basis of three concentric squares. The coordinates for the center area were from $(170,170)$ to $(340,340)$. The intermediate area ranged from $(85,85)$ to $(425,425)$, minus the center area, and the boundary area from $(0,0)$ to $(510,510)$, minus the center and intermediate areas.

To create the center-cluster version of a stimulus, each cluster was assigned to a randomly selected point within the center area, and the center of the cluster was located on that point, subject to the constraints that no point in a cluster fell outside of the center area or was closer than 30 pixels to a point in another cluster.

To create a single-edge-cluster version of a stimulus, the clusters in the center-cluster stimulus were treated as a single cluster and moved close to a boundary arc, following the procedure described in MacGregor (2013), subject to the additional constraint that the resulting $R$ value should approximate that of the corresponding center-cluster stimulus.

To create a multi-edge-cluster version of a stimulus, each cluster was assigned to a randomly selected point within the boundary area, and the center of a cluster was located on that point, subject to the constraints that all points in the cluster fell within the convex hull and no point was closer than 30 pixels to a point in another cluster or to a point on the convex hull.

The entire process was conducted five times in order to provide five replications of the four stimulus conditions. The mean $R$ values across the five stimuli in each condition were $1.13,0.98,1.01$, and 0.75 , for random, center clusters, singleedge clusters, and multi-edge clusters, respectively.

Procedure Testing was conducted individually, and each participant produced a TSP tour for all 20 stimulus point sets. The order of presentation of the stimuli was randomized. The stimuli were presented on screen, and participants responded by pointing and clicking. Participants were instructed to try to find the shortest tour connecting all of the points. An example was provided. At the end of the procedure, participants were asked to rank each of the four conditions from 1 to 4 , with 1 representing the easiest to solve and 4 the most difficult.

\section{Results and discussion}

The means and standard deviations for path length, solution time, and ease-of-completion rankings are provided in Table 1.

The length of the optimal tour was found for each of the 20 stimulus sets, and participants' tours were expressed in terms of PAO. Two participants appeared not to have understood or engaged in the task, producing many extremely long tours. In one case, the mean tour length was 4.5 standard deviations higher than that of the other participants, and in the other, 2.4 standard deviations higher. The data for these two participants were excluded from further analysis. For the remaining 18 participants, the tours had mean lengths (and standard deviations) of $3.6 \%(1.9 \%), 5.4 \%(2.6 \%), 3.9 \%(2.8 \%)$, and $1.1 \%$ $(0.9 \%)$ above optimal, for the random, center-cluster, singleedge-cluster, and multi-edge-cluster conditions, respectively. The corresponding mean solution times, in seconds (with standard deviations), were 53.5 (42.1), 47.6 (30.4), 47.2 (38.2), and $44.0(38.4) \mathrm{s}$. At the end of the procedure, participants ranked each of the four stimulus types for ease of completion (with 1 being easiest and 4 most difficult). The mean rankings (and standard deviations) were 3.28 (0.96), 2.97 (0.81), 2.14 (0.85), and 1.50 (0.69), for random, center clusters, singleedge clusters, and multi-edge clusters, respectively.

These results were analyzed using repeated measures analyses of variance (ANOVAs). For PAO, an overall significant difference emerged among the conditions, $F(3,51)=18.96$, $M S E=.006, p<.001, \eta^{2}=.53$. The differences among the conditions showed the following patterns (all $p$ values Bonferroni corrected): First, the multi-edge-cluster condition had significantly lower average PAO scores than did the other three conditions ( $p$ values $<.05$, Cohen's $d s=1.71,2.21$, and 1.34 , for comparisons with the random, center-cluster, and single-edge-cluster conditions, respectively). The difference between the center-cluster and the single-edge-cluster conditions was borderline significant ( $p=.06, d=0.56$ ). Finally, the center-cluster condition produced significantly longer tours than did the random condition $(p<.05, d=0.79)$. The differences between the pairs of conditions are illustrated in Table 1, using a combination of solid and dashed underlining to represent significant and borderline differences, respectively.

For solution times, an overall significant effect was also apparent, $F(3,51)=8.31, M S E=285.67, p<.001, \eta^{2}=.33$. In this case, the random condition had significantly longer response times than did the three cluster conditions (Bonferroni-corrected $p$ values $<.05, d=0.38,0.37$, and 0.56 for the comparisons with center clusters, single-edge clusters, and multi-edge clusters, respectively), whereas no other differences approached significance. Because nodes in the random condition tended to be farther, on average, from their nearest neighbors, it is possible that the significantly slower times reflected the greater movement times required to execute connections between points in that condition. Unfortunately, that issue cannot be answered with the present data.

Finally, for the ease-of-completion rankings there was an overall significant effect, $F(3,51)=12.92, M S E=.91$, $p<.001, \eta^{2}=.43$. Bonferroni comparisons indicated that the multi-edge-cluster condition and the single-edge-cluster condition were ranked as being significantly easier by participants than both the random and center-cluster conditions. The effect sizes for the multi-edge-cluster and single-edge-cluster comparisons with the random and center-cluster conditions were $d=2.13,1.96,1.25$, and 1.00 , respectively. 
Table 1 Means (and standard deviations) for percentage above the optimal length (PAO), solution time, and ease-of-completion rankings by stimulus condition, Experiment 1

\begin{tabular}{lllll}
\hline $\begin{array}{l}\text { Dependent } \\
\text { Variable }\end{array}$ & $\begin{array}{l}\text { Random } \\
(R=1.13)\end{array}$ & $\begin{array}{l}\text { Center } \\
(R=0.98)\end{array}$ & $\begin{array}{l}\text { Single-edge } \\
(R=1.01)\end{array}$ & $\begin{array}{l}\text { Multi-edge } \\
(R=0.75)\end{array}$ \\
\hline PAO & $3.6 \%(1.9 \%)$ & $\underline{5.4 \%(2.6 \%)}$ & $-.3 .9 \%(2.8 \%)$ & $\underline{1.1 \%(0.9 \%)}$ \\
Solution time & $\underline{53.5(42.1)}$ & $47.6(30.4)$ & $47.2(38.2)$ & $44.0(38.4)$ \\
Ranking & $3.28(0.96)$ & $2.97(0.81)$ & $\underline{2.14(0.85)}$ & $1.50(0.69)$
\end{tabular}

The primary purpose of the first experiment was to compare the effects on performance of $R$ value and cluster location. If $R$ value is the critical factor, we would expect the multi-edge condition, with the lowest $R$ value, to have the best performance. More tentatively (because of smaller $R$ differences), we might expect the two single-cluster conditions to show better performance than the random condition. These expectations were partially supported. First, the lowest- $R$ condition had significantly better PAO scores than the other conditions. Second, average completion times were faster on average in the lowest- $R$ condition, although significantly so only in comparison with the highest- $R$ condition. Third, participants ranked the lowest- $R$ condition as being easiest, on average, and significantly so in comparison with two of the three higher- $R$ conditions. However, contrary to expectations, the center-cluster condition, with the second lowest $R$ value, had significantly poorer PAO performance than did the random condition, which had the highest $R$ value. This result replicates a previous finding (MacGregor, 2013).

The results also partially supported the cluster location explanation, in which the expectation was that the two edge-cluster conditions would result in better performances (i.e., 4, $3>2$, 1). This expectation was wholly supported by participants' rankings (in which $4,3>2,1$ ), and partially supported by the PAO differences, in which $4>$ $3,2,1$ and the $3>2$ difference was borderline.

Unfortunately, because the experimental variables were not manipulated independently and the results partially supported both the $R$ value and cluster location explanations, they failed to provide a clear distinction between the two in accounting for performance differences. Consequently, a second experiment was conducted to better control for the effects of both factors.

\section{Experiment 2}

The second experiment was designed to vary $R$ value and the location of clusters independently.

\section{Method}

Participants The participants were 25 volunteers recruited from the campus community at the University of Victoria. Each was given a \$20 cafeteria voucher for participating.

Stimuli The stimuli for the high- $R$ condition were generated in a manner similar to those of Experiment 1. First, a 25-node point set was randomly generated to fall within a square with sides extending from 0 to 510 pixels, subject to the constraint that no point was closer than 15 pixels to another (points thus had an implicit radius of eight pixels).

Next, to generate the cluster conditions, all but the boundary points were removed from the random stimulus, leaving $h$ nodes on the convex hull. Then the numbers and sizes of clusters were randomly determined to provide from four or five clusters, each consisting of three to five nodes, such that the total number of nodes in clusters equaled $25-h$. Next, the relative positions of the nodes within each cluster were randomly determined, such that the minimum internode distance $\geq 5$ pixels and the maximum diameter of a cluster $\leq 65$ pixels $(2.5 \mathrm{~cm})$. The resulting means were 4.08 for cluster size, 4.33 for number of clusters, and 7.33 for number of nodes on the convex hull.

To locate clusters within the convex hull, the display was partitioned into three areas - center, intermediate, and boundary - on the basis of three concentric squares as in Experiment 1. The coordinates for the center area were from $(170,170)$ to $(340,340)$. The intermediate area ranged from $(85,85)$ to $(425,425)$, minus the center area, and the boundary area from $(0,0)$ to $(510,510)$, minus the center and intermediate areas.

To create a center-cluster version of a stimulus, each cluster was assigned to a randomly selected point within the center area, and the center of the cluster was located at that point, subject to the constraints that no point in a cluster fell outside the center area or was closer than 20 pixels to a point in another cluster, and that the $R$ value approximated 1 or greater. 
To create a single-edge-cluster version of a stimulus, the clusters in the center-cluster stimulus were treated as a single cluster and moved close to a boundary arc, following the procedure described in MacGregor (2013), subject to the additional constraint that the resulting $R$ value approximated that of the corresponding center-cluster stimulus.

To create a multi-edge-cluster version of a stimulus, each cluster was assigned to a randomly selected point within the boundary area, and the center of a cluster was located on that point, subject to the constraints that all points on the cluster fell within the convex hull, no point was closer than 20 pixels to a point in another cluster or than 30 pixels to a point on the convex hull, and the overall $R$ value approximated those of the other conditions.

The entire process was conducted three times to provide three replications of each of the three high- $R$ stimulus conditions. The mean $R$ values across the three stimuli in each condition were $1.23,1.24$, and 1.10 , for the center-cluster, single-edge-cluster, and multi-edge-cluster conditions, respectively.

The stimuli in the high- $\mathrm{R}$ conditions were used to generate corresponding stimuli in the low- $R$ conditions. In doing so, it was important to control other factors that might influence performance - namely, the configuration of the clusters and the number of nodes on the convex hull. The only means of reducing $R$ values while holding these other factors constant was to move the hull points closer together, which was done by sliding a randomly selected hull point along a connecting arc toward one of its two neighboring hull nodes. In doing so, a distance of at least 20 pixels was preserved between all adjacent hull nodes. The objective was to reduce the stimulus's $R$ value to 0.70 or lower, and if moving one hull point did not achieve this, additional hull points were randomly selected and manipulated in the same way. At the end of this process, the mean $R$ values across the nine stimuli in the low- $R$ condition were $0.69,0.61$, and 0.62 , for the center-cluster, single-edge-cluster, and multi-edge-cluster stimuli, respectively. The mean overall $R$ values for the stimuli in the highand low- $R$ conditions were 1.19 and 0.64 , respectively. The stimuli are illustrated in Fig. 2.

Procedure The procedure followed that of the first experiment.

\section{Results and discussion}

Path length Optimal tours were found for each of the 18 stimulus point sets, and the participants' tours were expressed in terms of PAO. Three participants did not appear to comply with the task requirements, producing many long tours with multiple crossed arcs. In all three cases, the mean PAO was more than four standard deviations higher than that of the remaining participants. The data for these three participants were excluded from further analysis. For the remaining 22 participants, the tours had mean lengths (and standard deviations) of $9.9 \%(6.5 \%), 3.7 \%(3.2 \%)$, and $4.1 \%(4.6 \%)$ above optimal, for the center-, single-edge-, and multi-edge-cluster conditions, respectively in the high- $R$ condition. For the low- $R$ condition, the corresponding results were $5.0 \%(2.7 \%), 4.7 \%$ $(3.3 \%)$, and $4.1 \%(3.2 \%)$. The results are shown in the left panel of Fig. 3, where the columns represent high- $R$ scores minus low-R scores, and error bars represent $95 \%$ confidence intervals for the differences in means.

These results were analyzed using a repeated measures $2 \times 3$ ANOVA, which indicated a significant main effect of cluster location, $F(2,42)=17.85, M S E=.001, p<.001, \eta^{2}=.46$, and a borderline significant effect of $R$ value, $F(1,21)=4.09$, $M S E=.001, p=.06, \eta^{2}=.16$. A significant interaction effect between cluster location and $R$ value was also apparent, $F(2,42)=11.10, M S E=.001, p<.001, \eta^{2}=.35$. As may be seen from Fig. 3, the effect of $R$ value was limited to the center-cluster condition only, in which the PAO was twice as great in the high- as in the low- $R$ condition. Bonferroni-corrected tests of simple main effects indicated that PAO was significantly higher $(p<.05)$ for the high- $R$ condition than for the low- $R$ condition for the center cluster location only. No other differences approached significance. The results suggest that when clusters are located centrally, performance is poorer than when the same clusters are located at either a single edge or at multiple edges, but for high $R$ values only. At low $R$ values, neither $R$ value nor cluster location mattered, and performances were equally good across the remaining conditions.

Solution time The mean solution times (and standard deviations) were of 60.31 (23.78), 43.53 (15.34), and 48.22 (19.42) $\mathrm{s}$ for the center-cluster, single-edge-cluster, and multi-edgecluster conditions, respectively, in the high- $R$ condition. For the low- $R$ condition, the corresponding results were 48.00 (18.99), 46.79 (19.09), and 45.93 (16.35) s. The results are shown in the right panel of Fig. 3, where the columns represent high- $R$ scores minus low- $R$ scores, and error bars represent $95 \%$ confidence intervals for the differences in means.

Again, the results were analyzed using a $2 \times 3$ repeated measures ANOVA, which indicated significant effects of cluster location, $F(2,42)=14.11, M S E=70.10, p<.001, \eta^{2}=.40 ; R$ value, $F(1,21)=6.16, M S E=76.63, p=.02, \eta^{2}=.23$; and their interaction, $F(2,42)=5.72, M S E=119.75, p<.01$, $\eta^{2}=.21$. It may be seen from the right panel of Fig. 3 that the results followed a similar pattern to those for PAO, and tests of the simple main effects of $R$ at each level of cluster location showed that solution times were significantly longer $(p<.05)$ for the high- $R$ condition for the center-cluster condition only.

Solution ratings At the end of the procedure, participants were asked to rank the center clusters, single-edge clusters, and multi-edge clusters for ease of completion (with 1 being 
HIGH-R STIMULI

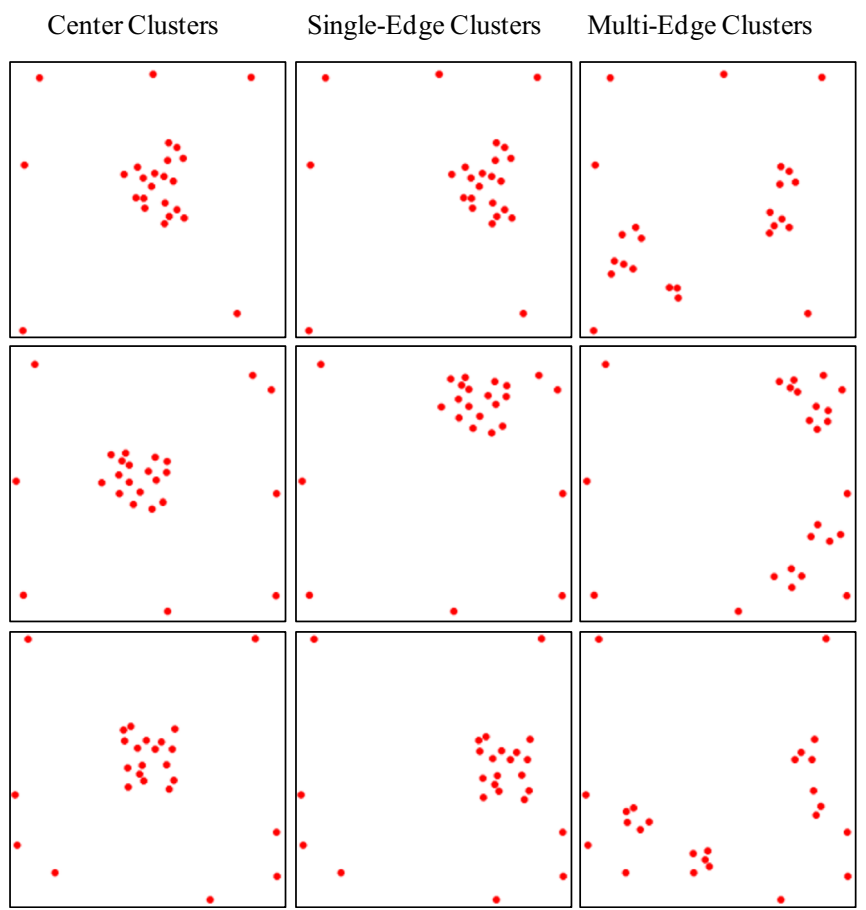

LOW-R STIMULI

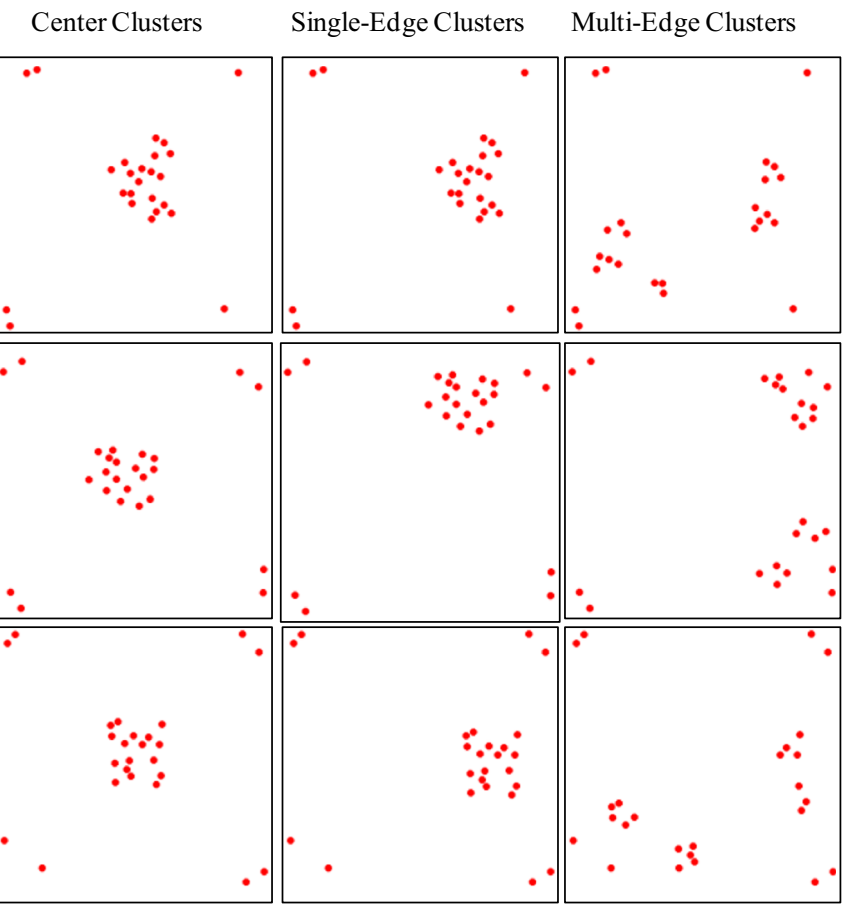

Fig. 2 Stimuli for Experiment 2, with high- $R$ stimuli in the left block, low- $R$ stimuli in the right, and within each block, center clusters, single-edge clusters, and multi-edge clusters in the first, second, and third columns, respectively

easiest and 3 being most difficult). The mean rankings (and standard deviations) were $2.57(0.75), 1.86(0.57)$, and 1.52 $(0.75)$ for the center, single-edge, and multi-edge clusters, respectively. There was an overall significant difference among the conditions, $F(2,40)=8.40, M S E=.72, p<.001, \eta^{2}=.30$. Paired comparisons indicated that both of the edge cluster conditions were ranked as being significantly easier than the center cluster condition (Bonferroni-corrected $p \mathrm{~s}<.05$, $d \mathrm{~s}=1.07$ and 1.20).

In sum, the results of the second experiment provided a relatively consistent pattern in which performance was affected by both cluster location and cluster density, as measured by $R$ value. However, the two factors interacted, so that only the combination of centrally located clusters and high $R$ values resulted in poorer performance. When clusters were located at a single boundary edge or at multiple edges, performances were equally good for both high and low $R$ values, whereas for the low- $R$-value stimuli, cluster location had no significant effect on performance. Nevertheless, participants appeared to be influenced by the fact that some stimuli with centrally located clusters were more challenging, and they ranked central-cluster stimuli as being more difficult than the others.

\section{General discussion}

This article has examined the effects of the location and distribution of dot clusters on participants' TSP performance. Most models of TSP performance involve a stage of processing at which point sets are organized into perceived clusters

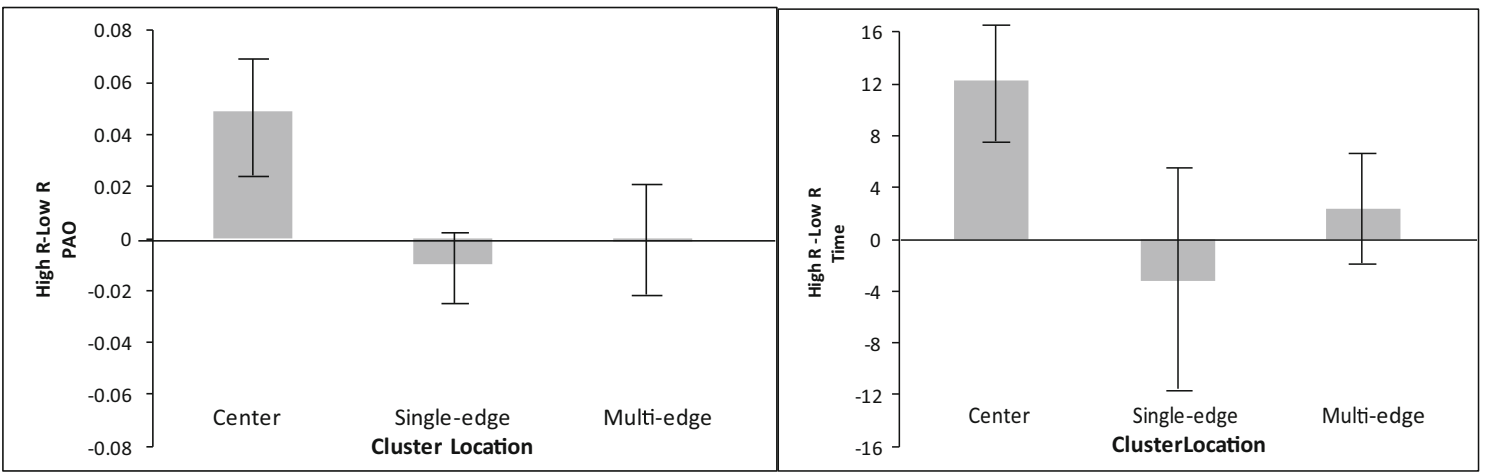

Fig. 3 Results from Experiment 2 for differences in solution lengths (as percentages above optimal [PAO]), on the left, and in solution times, on the right 
(Best, 2005; Kong \& Schunn, 2007; Pizlo et al., 2006), on the basis of either hierarchical (Best, 2005; Pizlo et al., 2006) or k-means (Kong \& Schunn, 2007) clustering, and it has been hypothesized that the presence of clusters facilitates performance (Dry et al., 2012). However, the results of previous experimental tests of the hypothesis have been equivocal. Dry et al. (2012) used dot arrays corresponding to clustered, random, and regular distributions, as measured by the Clark-Evans index of aggregation $(R)$, and found evidence of better performance with clustered than with random or regular distributions. However, in manipulating the distribution of dot clusters, the locations of dot clusters may have varied also (MacGregor, 2013).

The present research reported two experiments designed to unravel the effects of $R$ and cluster location. The first experiment used several combinations of degree of clustering and cluster location, and the second varied both variables independently.

The results of the first experiment provided partial support for the hypothesis of better performance with clustered point sets, in that performance was superior in the condition with the lowest $R$ value. However, performance in the random condition, with the highest $R$ value, was significantly better than that in the center-cluster condition, which was not consistent with the hypothesis that clustering facilitates TSP performance.

The results of the second experiment further clarified the roles of cluster distribution and cluster location by finding that they interact. When $R$ value was low, the location of a large cluster did not matter, and performance, in terms of PAO, was equally good across all three types of cluster locations. However, when $R$ value was high, locating the cluster centrally caused performance to deteriorate significantly.

Several limitations should be acknowledged, involving the somewhat artificial nature of the stimuli, which arose in part from the procedures used to manipulate cluster location and $R$ value. One concern is that the clusters perceived by participants may not have corresponded to the clusters intended in the process of stimulus production. A second is that the procedure required to obtain the low- $R$-value stimuli, by moving hull points closer, although it reduced $R$ mathematically, may not have similarly increased the perception of clusters. Two steps were taken to further assess the potential impacts of these concerns. First, a simulation was conducted to investigate potential cluster formation, using the stimuli from both experiments. Second, participants' tours were analyzed for consistency with both the intended clusters and the clusters produced by the simulations.

\section{Simulation of clusters}

The simulations were based on hierarchical cluster analyses using the $x$-and $y$-coordinates of the stimulus point sets as the data. Hierarchical clustering was used because TSP clustering models are typically based on a hierarchical approach (Best, 2005; Pizlo et al., 2006). The analyses used the SPSS hierarchical-clustering procedure, which employs an agglomerative method. Best's procedure, which is based on the CODE model, also employs a bottom-up approach (Compton \& Logan, 1993). The results reported here are from the first stage of the analysis, representing the lowest level of clustering. The simulations of the first-experiment stimuli provided several relevant outcomes. One was that the total number of points included in clusters proved to be the best single "perceptual" factor related to $R$ value. The correlation between $R$ value and the number of points in clusters across the 20 stimuli was $r=-.85, p<.05$, indicating that the lower the $R$ value (i.e., the greater the overall indication of clustering), the more points were included in clusters (or the fewer were the number of isolates). The relationship held both for the random stimuli $(r=-.92)$ and the clustered stimuli $(r=$ -.85 ). Thus, although the clustered stimuli were artificially constructed, they nevertheless appeared to preserve a potentially important relationship between a mathematical and a "perceptual" measure of clustering.

A second relevant finding is illustrated in Fig. 4, which compares the clusters produced by the cluster analyses (bounded by solid ellipses) with those intended in the construction of the stimuli (bounded by dashed ellipses). The results were similar for the stimuli from both experiments and, for simplicity, are reported here using the Experiment 2 stimuli only. As may be seen from the figure, the simulated clusters produced by the cluster analysis were generally consistent with the intended central, single-edge, and multi-edge locations of clusters. In some cases, the simulated and intended clusters coincided exactly, but when this was not the case, the simulated clusters included more points.

To further compare the simulated with the intended clusters, participants' responses were assessed for conformity with both. In doing so, a count was made for each cluster of the maximum number of points that a tour visited within the cluster before moving beyond the cluster. The starting and ending points within a cluster were counted, so that the maximum possible "cluster conformity" equaled the size of a cluster. Summed over the 22 participants' responses to the 18 point sets, the frequency of connections conforming to the simulated clusters was 7,884 of a possible 8,475 (93.0\%), and the corresponding frequency conforming to the intended clusters was 6 , 455 of a possible $6,979(92.5 \%)$. (The simulations produced more clusters than intended, which accounts for the larger totals.) On the basis of these comparisons, participant responses appear to have conformed highly with both the intended and the simulated clusters. The finding is consistent with participants having responded to the clusters as planned.

There remain concerns that the $R$ value in the low- $R$ condition was artificially lowered by moving several hull points 
HIGH-R STIMULI

Center Clusters

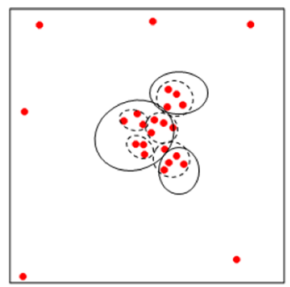

Single-Edge Clusters

Multi-Edge Clusters

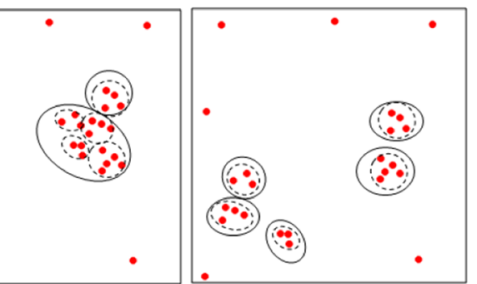

Center Clusters

LOW-R STIMULI

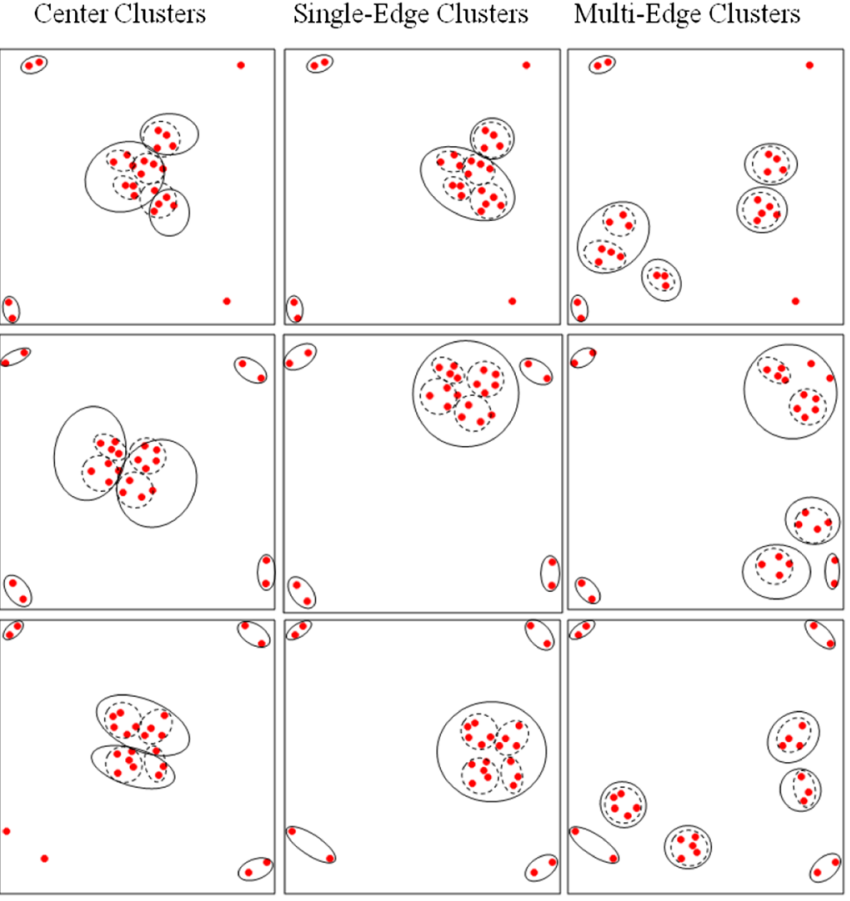

Fig. 4 Intended clusters (dashed ellipses) and clusters from a hierarchical cluster analysis of the Experiment 2 stimuli (solid ellipses)

close together and that the resulting $R$ may not have been indicative of the overall degree of perceptual clustering. Figure 4 also indicates that the clustering of hull points occurred naturally as well as through the manipulation of $R$ value. Across the 18 stimuli, a total of 20 hull points were included in clusters before the manipulation that moved a hull point into the proximity of another. The artificial clusters produced by moving hull points together presumably contributed to $R$ value in the same way as these naturally occurring clusters involving hull points, although whether this is a desirable property of the $R$ measure may be questioned. A potential concern therefore remains as to how representative the present stimuli may be of the range of $R$ values they were intended to reflect, and future research may find better methods for manipulating $R$.

Beyond the issues raised by the simulations, several other limitations relate to potentially uncontrolled factors that may have affected performance. One is the number of possible "indentations," and another is the number of possible intersections in the stimuli. An indentation occurs whenever a TSP path connects interior points between two adjacent points on the convex hull, and the total number possible is determined by the number of hull points or the number of interior points, whichever is fewer. Previous research has indicated that human solutions show a preference for fewer indentations, and that this is independent of the optimality of the solution (MacGregor, 2012). In the present case, because the numbers of hull and interior points were held constant within each family of stimuli, the number of possible indentations was controlled for. The number of potential intersections also has been found to affect performance, and also to increase as a function of $R$ (Dry et al., 2012). Because of the latter relationship, a concern is that the high- $R$ stimuli may have contained more potential intersections than the low- $R$ stimuli. However, because performance improves with increasing number of intersections (Vickers et al., 2003), any effect would have tended to provide an uncontrolled advantage to the high- $R$ stimuli and to run counter to the experimental hypotheses tested here. Nevertheless, differences in the numbers of intersections may have been a factor in the finding in the first experiment, in which performance in the higher- $R$ random condition was superior to that of the lower- $R$ center-cluster condition.

However, within these limitations, the results have implications for TSP research, by adding to the array of stimulus factors that have already been identified as affecting performance. Previous research has demonstrated effects due to the number of points (Dry et al., 2006; Graham et al., 2000), the number of nonhull points (MacGregor \& Ormerod, 1996), the number of potential intersections (Vickers et al., 2003), and the number of "indentations" (MacGregor, 2012). The present research consolidates and further clarifies the roles of degree of clustering and the location of clusters and, by doing so, further supports the attention to cluster formation in particular, and to perceptual factors in general, that a number of theoretical approaches have emphasized (Best, 2005; Kong \& Schunn, 2007; Pizlo et al., 2006; Vickers et al., 2003). 
Author note The research was supported by a research grant from the National Science and Engineering Research Council of Canada.

\section{References}

Applegate, D. L., Bixby, R. E., Chvátal, V., \& Cook, W. J. (2006). The traveling salesman problem: A computational study. Princeton, $\mathrm{NJ}$ : Princeton University Press.

Asano, T., Bhattacharya, B., Keil, M., \& Yao, F. (1988). Clustering algorithms based on minimum and maximum spanning trees. In Proceedings of the Fourth Annual Symposium on Computational Geometry (SCG '88) (pp. 252-257). New York, NY: ACM. doi: $10.1145 / 73393.73419$

Best, B. J. (2005). A model of fast human performance on a computationally hard problem. In B. G. Bara, L. Barsalou, \& M. Bucciarelli (Eds.), Proceedings of the 27th Annual Conference of the Cognitive Science Society (pp. 256-261). Mahwah, NJ: Erlbaum.

Bland, R. E., \& Shallcross, D. F. (1987). Large traveling salesman problem arising from experiments in x-ray crystallography: A preliminary report on computation (Technical Report No. 730). Ithaca, NY: Cornell University, School of Operations Research and Information Engineering.

Clark, P. J., \& Evans, F. C. (1954). Distance to nearest neighbor as a measure of spatial relationships in populations. Ecology, 35, 445-453.

Compton, B. J., \& Logan, G. D. (1993). Evaluating a computational model of perceptual grouping by proximity. Perception \& Psychophysics, 53, 403-421. doi:10.3758/BF03206783

Dallari, F., Marchet, G., \& Ruggeri, R. (2000). Optimisation of man-onboard automated storage/retrieval systems. Integrated Manufacturing Systems, 11, 87-93.

Dry, M. J., Lee, M. D., Vickers, D., \& Hughes, P. (2006). Human performance on visually presented traveling salesperson problems with varying numbers of nodes. Journal of Problem Solving, 1, 20-32.

Dry, M., Preiss, K., \& Wagemans, J. (2012). Clustering, randomness, and regularity: Spatial distributions and human performance on the traveling salesperson problem and minimum spanning tree problem. Journal of Problem Solving, 4(1), 2. Available at http://docs.lib.purdue.edu/jps/vol4/iss1/2.

Garey, M. R., \& Johnson, D. S. (1979). Computers and intractability: A guide to the theory of NP-completeness. San Francisco, CA: W. H. Freeman.

Gärling, T. (1989). The role of cognitive maps in spatial decisions. Journal of Environmental Psychology, 9, 269-278.

Golden, B., Bodin, L., Doyle, T., \& Stewart, W. (1980). Approximate traveling salesman algorithms. Operations Research, 28, 694-711.

Graham, S. M., Joshi, A., \& Pizlo, Z. (2000). The traveling salesman problem: A hierarchical model. Memory \& Cognition, 28, 11911204. doi:10.3758/BF03211820

Hill, A. V. (1982). An experimental comparison of human schedulers and heuristic algorithms for the traveling salesman problem. Journal of Operations Management, 2, 215-223.

Hirtle, S. C., \& Gärling, T. (1992). Heuristic rules for sequential spatial decisions. Geoforum, 23, 227-238.

Johansson, G. (1973). Visual perception of biological motion and a model for its analysis. Perception \& Psychophysics, 14, 201-211. doi:10. 3758/BF03212378

Julesz, B. (1965). Texture and visual perception. Scientific American, $212,38-48$

Kong, X., \& Schunn, C. D. (2007). Global versus local information processing in visual/spatial problem solving: The case of traveling salesman problem. Cognitive Systems Research, 8, 192-207.
Krolak, P., Felts, W., \& Marble, G. (1971). A man-machine approach toward solving the traveling salesman problem. Communications of the ACM, 14, 327-334.

Lawler, E. L., Shmoys, D. B., Rinnooy Kan, A. H. G., \& Lenstra, J. K. (1985). The traveling salesman problem. New York, NY: Wiley.

Lee, M. D., \& Vickers, D. (2000). The importance of the convex hull for human performance on the traveling salesman problem: A comment on MacGregor and Ormerod (1996). Perception \& Psychophysics, 62, 226-228. doi:10.3758/BF03212074

MacGregor, J. N. (2012). Indentations and starting points in Traveling Sales Tour Problems: Implications for theory. Journal of Problem Solving, 5(1), 3. doi:10.7771/1932-6246.1140

MacGregor, J. N. (2013). Effects of cluster location on human performance on the Traveling Salesperson Problem. Journal of Problem Solving, 5(2), 3. doi:10.7771/1932-6246.1151

MacGregor, J. N., \& Chu, Y. (2011). Human performance on the traveling salesman and related problems: A review. Journal of Problem Solving, 3(2), 2. Available at http://docs.lib.purdue.edu/jps/vol3/ iss $2 / 2$.

MacGregor, J. N., \& Ormerod, T. (1996). Human performance on the traveling salesman problem. Perception \& Psychophysics, 58, 527-539. doi:10.3758/BF03213088

MacGregor, J. N., Ormerod, T. C., \& Chronicle, E. P. (1999). Spatial and contextual factors in human performance on the traveling salesman problem. Perception, 28, 1417-1427.

O'Callaghan, J. F. (1974). Human perception of homogeneous dot patterns. Perception, 3, 33-45.

Ormerod, T. C., \& Chronicle, E. P. (1999). Global perceptual processing in problem solving: The case of the traveling salesperson. Perception \& Psychophysics, 61, 1227-1238. doi:10.3758/BF03207625

Pickett, R. M. (1964). The perception of a visual texture. Journal of Experimental Psychology, 68, 13-20.

Pizlo, Z., Stefanov, E., Saalweachter, J., Haxhimusa, Y., \& Kropatsch, W. G. (2006). Traveling salesman problem: A foveating pyramid model. Journal of Problem Solving, 1, 83-101.

Polivanova, N. I. (1974). On some functional and structural features of the visual-intuitive components of a problem-solving process. Voprosy Psikhologii [Questions of Psychology], 4, 41-51.

Pomerantz, J. R. (1981). Perceptual organization and information processing. In M. Kubovy \& J. R. Pomerantz (Eds.), Perceptual organization (pp. 141-180). Hillsdale, NJ: Erlbaum.

Ramachandran, V. S., \& Anstis, S. M. (1986). The perception of apparent motion. Scientific American, 254, 102-109.

Sangalli, A. (1992). Why sales reps pose a hard problem. New Scientist, 12, 24-28.

Trick, L. M., \& Enns, J. T. (1997). Clusters precede shapes in perceptual organization. Psychological Science, 8, 124-129.

Vickers, D., Butavicius, M. A., Lee, M. D., \& Medvedev, A. (2001). Human performance on visually presented traveling salesman problems. Psychological Research, 65, 34-45.

Vickers, D., Lee, M. D., Dry, M., \& Hughes, P. (2003). The roles of the convex hull and the number of potential intersections in performance on visually presented traveling salesperson problems. Memory \& Cognition, 31, 1094-1104. doi:10. 3758/BF03196130

Vickers, D., Mayo, T., Heitmann, M., Lee, M. D., \& Hughes, P. (2004). Intelligence and individual differences in performance on three types of visually presented optimization problems. Personality and Individual Differences, 36, 1059-1071.

Vickers, D., Lee, M. D., Dry, M., Hughes, P., \& McMahon, J. A. (2006). The aesthetic appeal of minimal structures: Judging the attractiveness of solutions to traveling salesperson problems. Perception \& Psychophysics, 68, 32-42. doi:10.3758/ BF03193653 
Wertheimer, M. (1950). Laws of organization in perceptual forms. In W. D. Ellis (Ed.), A source book of Gestalt psychology (pp. 71-88). New York, NY: Humanities Press (Original work published 1923).
Zahn, C. Y. (1971). Graph-theoretical methods for detecting and describing Gestalt clusters. IEEE Transactions on Computers, $20,68-86$. 